\title{
PERCEPCIÓN DE LOS DOCENTES EGRESADOS DE FÍSICA SOBRE EL IMPACTO DE LAS DIFERENTES MATERIAS CURSADAS EN LA CARRERA
}

\author{
Physics teacher's perception about the impact of the \\ different subjects studied in their career.
}

\author{
Alejandro Parrella \\ Administración Nacional de Educación Pública \\ Consejo de Formación en Educación. Cerp del Este, Uruguay \\ ORCID 0000-0002-5626-6057 \\ alejandroparrella@gmail.com
}

Recibido 26 de abril de 2019

Aceptado 19 de julio de 2019

\section{Resumen}

En este artículo se desarrolla una indagación realizada a comienzos de 2019 que tuvo por objetivo establecer pautas para confeccionar un índice de impacto de las asignaturas aprobadas en la carrera de profesor de Física del plan $2008^{1}$ a partir de la valoración de los egresados recientes. Para ello se recurrió a encuestar a profesores de Física, egresados de todo el Uruguay de los últimos 5 años. La encuesta fue online, anónima, en la que terminaron participando 71 personas, superando la mitad de los egresados de ese lapso. En esa encuesta, se pedía la valoración de las diferentes asignaturas cursadas durante el profesorado, en relación al aporte de esa asignatura, en algún aspecto, a su carrera como docente, principalmente para sus primeros años de trabajo como profesor. El propósito de esta encuesta no es categorizar la pertinencia de las asignaturas en formación docente en función de las respuestas de los egresados, sino establecer, a partir de su valoración, pautas para confeccionar un índice de presencia de los estudiantes de profesorado de Física a cargo de los cursos de esta asignatura en la enseñanza media, de acuerdo a las asignaturas aprobadas en la carrera.
\end{abstract}

Palabras clave Evaluación de asignaturas, índice de impacto, estudiantes de profesorado,

\begin{abstract}
In this article is developed a research carried out at the beginning of 2019 that aimed to establish guidelines to make an impact index of the subjects passed in the career of Physics teacher degree of the $2008^{2}$ syllabus, based on the assessment of recent graduates. For this purpose, there were interviewed physics teachers, graduates for the last 5 years, from all along Uruguay. The survey was online, anonymous, where 71 people end up participated, outranging half of the graduates of that period. In this survey, the assessment of the different subjects of the sylabus taken during the career was required, related to the contribution of that subject, in some aspect, mainly for his first years of working as a teacher. The purpose of this survey isn ' $t$ to assess the relevance of the subjects in the career according to the answers of the recent graduates, but from this answers it is sought to elaborate an impact index of the presence of the students of Physics Teachers degree in charge of the courses of secondary education.
\end{abstract}

Key words Subject evaluation, impact index, college students.

\section{Introducción}

1 Plan 2008 de Formación de Profesores del Consejo de Formación en Educación (C.F.E.). Administración Nacional de Educación Pública(A.N.E.P.) - Uruguay..

22008 Syllabus tocgetcthec Physicsc Teacherc Degree in the Consejo de Formación en Educación (C.F.E.).Administración Nacional de Educación Pública (A.N.E.P.) - Uruguay. 
La titulación oficial de los profesores de Física para la enseñanza media es, a la vez, la meta y el problema crónico. Meta en tanto propósito de sucesivas políticas educativas, de autoridades y legisladores; y problema crónico por ser Física una de las asignaturas con peores guarismos históricos. Luego de más de dos décadas de diferentes impulsos en políticas de formación docente, en la actualidad la tasa de docentes titulados de Física es en el entorno del $60 \%{ }^{3}$. Esto lleva a la necesidad de cubrir el resto de los puestos docentes con otras personas (para efectos de este artículo se denominarán “idóneos”). Varias décadas atrás, cuando la enseñanza media era solo para un grupo selecto de estudiantes, en Montevideo la idoneidad era cubierta frecuentemente por ingenieros $\mathrm{u}$ otros profesionales afines, quienes dictaban las clases de Física. En otras localidades, la suerte era dispar. No siempre el idóneo era tal.

En la actualidad, factores como la expansión de la cobertura de la enseñanza media, el aumento de la matrícula de profesorado y la progresiva convicción de que la profesión docente es una profesión compleja que no solo requiere de conocer el campo disciplinar, ha llevado que, para el ingreso a cubrir las vacantes en las aulas de enseñanza media, se priorice a quienes ya han comenzado su carrera de profesorado de Física. Como ejemplo de ello, notar que en el ámbito del Consejo de Educación Secundaria (C.E.S.) no solo los dos últimos concursos de efectividad han sido exclusivos para egresados de formación docente, sino que para integrar la nómina de aspirantes interinos se prioriza la aprobación de los cursos de la carrera docente específica por sobre otra formación afín. (C.E.S., 2018). Como consecuencia de esta situación, la enorme mayoría de los estudiantes de profesorado trabaja como docente antes de egresar. ${ }^{4}$ Esto genera un doble desafío: no solo se debe investigar sobre los estudiantes de profesorado en su rol de docentes de enseñanza media, para poder comprender las características de su inserción en los liceos y su impacto en estas instituciones, sino que las políticas de formación docente no deben ver al estudiante de profesorado que dicta clases como un "problema" o un "handicap". A modo de ejemplo, declaraciones de Ana Lopater, Directora General del Consejo de Formación en Educación (C.F.E.), indica como causa del rezago o abandono de la carrera de algunos estudiantes que "muchas veces, algunos alumnos dejan un tiempo y luego retoman, en parte vinculado a la demanda de docentes, lo cual deriva en que comiencen a dar clase antes de recibirse, sobre todo en materias como: Inglés, Idioma Español, Física, Química y Matemática" (Presidencia, 2017). Los estudiantes de profesorado de Física, en su rol de docentes de enseñanza media no son hoy un "problema", sino una realidad desde donde trabajar. Esa realidad, a corto y mediano plazo, no tiene perspectivas de modificarse.

En el escenario que se acaba de describir, los estudiantes de profesorado que comienzan a ejercer como docentes lo hacen en las instituciones más desfavorecidas. Como ejemplo, se rescatan los testimonios de estudiantes del profesorado semipresencial de Tacuarembó (Parrella, 2018), observándose que sus experiencias docentes se situaban en localidades pequeñas y alejadas como Achar, Ansina,

3 El porcentaje difiere un poco si se contabiliza por docente o por horas de clase. Como los docentes egresados tienden a tener cargas horarias mayores, los porcentajes de horas docentes cubiertas por profesores titulados es mayor al cociente entre docentes titulados/ total de docentes. Diferentes censos públicos miden uno u otro dato, por lo que se opta por escribir "en el entorno del 60\%".

4 Esta afirmación se corroborará, una vez más, con cifras cuantitativas en esta misma indagación.

Avances en la Enseñanza de la Física 
San Gregorio de Polanco o en la Cárcel. Cuando estas comunidades incorporan un estudiante de profesorado a su plantilla de docentes, es indudablemente una mejora, porque antes de ese estudiante a cargo de las clases había probablemente una persona sin formación específica.

Va de suyo que el objetivo de una política educativa de formación docente es propender a que todos los docentes tengan la titulación correspondiente, pero en la realidad actual la presencia de los estudiantes de profesorado en los liceos no solo se observa como mejora como se detalló anteriormente, sino que además puede servir como ejemplo para aquellos que están ejerciendo y no tienen formación, para que los impulse a comenzar su formación específica. Sobre el particular, se coincide con Klein $(2015,427)$ cuando plantea que "debería estimulase que todos los interinos pasen a ser estudiantes", promoviendo la formación específica, con acciones como "reorganización de las categorías (de interinos), "premios" para aquellos que estudien, asignación de horas de laboratorio para tener horas extra aula, considerar su condición de estudiante a la hora de la confección de los horarios de trabajo, brindándole una distribución coherente con los horarios de los IFPF. ${ }^{5}$ En contrapartida, mantenimiento de estas "fácilidades" siempre que se observe un avance claro en la carrera en Física". $(2015,428)$

Por otro lado, es constatable que los estudiantes no van aprobando las diferentes asignaturas que componen el profesorado en forma lineal, ni van completando progresivamente cada ciclo (año lectivo), sino que se observan diferentes velocidades en los tres pilares de la formación docente: núcleo común, asignaturas específicas y didáctica. Los estudiantes van eligiendo las materias como parte de su estrategia de carrera. Es allí donde se establece la necesidad de ponderar las materias que esos estudiantes que se desempeñan como docentes, fueron aprobando. ¿Cuánto impacta en sus prácticas como docentes de liceo las materias que eligen para ir avanzando en su carrera? ¿Todas impactan por igual? Para realizar una valoración adecuada, se entiende que se requiere recabar sistemáticamente la opinión de los egresados recientes. Estos profesores tienen más presente el impacto de cada materia en la mejora de su práctica docente debido a su cercanía temporal con la cursada.

Esta misma búsqueda de información, a su vez permite obtener datos sobre los propios cursos de formación docente. En ese sentido, se desea dejar constancia que no es propósito de este trabajo categorizar la pertinencia de las asignaturas en formación docente en función de las respuestas de los egresados. Las categorizaciones obtenidas pueden dar lugar a nuevas preguntas que deben ser parte de indagaciones más profundas, de corte cualitativo. Realizadas estas aclaraciones, se define que el objetivo de este trabajo es establecer pautas para confeccionar un índice de presencia de los estudiantes de profesorado de Física a cargo de los cursos de esta asignatura en la enseñanza media, en función de las asignaturas aprobadas en la carrera a partir de la valoración de los egresados recientes.

\section{Metodología}

Para la elaboración de este índice se realizó una encuesta con los egresados de todo el país de los últimos 5 años. El número de profesores que se titularon en ese lapso es del orden de 120. La encuesta fue online, anónima, a la que se accedía a través de un enlace proporcionado en un mensaje de presentación. Ese mensaje

$5 \quad$ En el original la sigla IFPF refiere a Institutos de Formación de Profesores de Física.

6 En el original dice "fáciles", constatándose claramente que se trata de un error de tipeo. 
fue enviado a 100 de ellos, de los cuales 71 respondieron a la encuesta, en forma voluntaria, contactados por el investigador, ya sea por Whatsapp o por correo electrónico. Llamó la atención la diferencia entre la tasa de respuesta según el medio de contacto, pues por correo electrónico, la tasa de respuesta fue del $57 \%$, mientras que para los mensajes enviados por Whatsapp, la tasa de respuesta, y posterior llenado de la encuesta, ascendió a 77\%.

El cuestionario fue testeado previamente con otros profesores egresados más experientes, a los que se les pidió que hicieran comentarios además de llenar la encuesta. Esto permitió hacer ajustes a las consignas y estimar adecuadamente el tiempo de llenado de la misma. Las respuestas de los profesores indagados comenzaron a tener tendencias firmes a partir del encuestado número 20 , habiéndose hecho otra observación a las 45 respuestas, ambas congruentes entre sí y con el resultado final. Se tuvo particular cuidado de encuestar a egresados de todos los centros de formación del país en los que se dicta la carrera de Profesor de Física, de modo de que a posteriori no se pueda atribuir un alto o bajo índice de una materia en particular al efecto de buena o mala práctica docente puntual. La dispersión de respuestas observada en la mayoría de las materias no resultó alta, salvo un caso excepcional de Espacio Interdisciplinario (E.C.I.), que se discute específicamente en el apartado de resultados.

La redacción de la pregunta es de particular importancia para no generar malentendidos, sobre todo en el propósito de la indagación, por lo que se opta por transcribirla aquí:

La siguiente es una encuesta anónima en el marco de una investigación sobre los estudiantes de profesorado que trabajan como docentes en el sistema público antes de egresar. Una de las variables que se presentan en esta situación es la cantidad y el tipo de asignaturas que cada estudiante ha aprobado en su carrera al momento de tomar grupos a cargo. Por eso se plantea esta encuesta a quienes han aprobado todas las materias, para conocer su visión de cómo lo aprendido en esas materias incidió en sus primeros años de desempeño como docente. El proceso de respuesta de este cuestionario lleva aproximadamente entre 10 y 15 minutos.

En la primera parte se presentan una lista de asignaturas que Ud. aprobó en el transcurso de su carrera para obtener el título de profesor de Física. Allí valore de 0 a 5 (donde 0 es el puntaje mínimo, 5 el puntaje máximo) cuanto cree que lo que aprendió en cada materia, le aportó, en algún aspecto, a su carrera como docente, principalmente para sus primeros años de trabajo como profesor. Si no la cursó, o no tiene recuerdos de esa materia, déjela sin responder. Al final, en caso de ser necesario, hay un espacio optativo para realizar las aclaraciones que crea conveniente sobre alguna respuesta. En la segunda parte, hay 4 preguntas breves sobre variables personales (año de ingreso a formación docente, de egreso, etc).

Vale decir que no hubo consultas de los encuestados en relación a la comprensión de la consigna. Las escasas consultas referían a qué hacer en caso de haber revalidado alguna materia, o en algún caso de no haberla cursado por haber hecho cambio de plan. 
Para este apartado se realizará primero un análisis general de las valoraciones obtenidas. En una segunda parte, al mirar con detenimiento algunos guarismos, se harán comentarios en detalle.

Vale aquí aclarar que algunos encuestados no solo participaron generosamente de la indagación, sino que además encontraron un canal para expresar cosas que particularmente les interesaban. En un caso, un participante respondió al mensaje del investigador diciendo "Encuesta realizada!! Muchas gracias por permitirme participar. Es la primera vez. que alguien me pregunta sobre las materias que realmente pesaron en mi carrera y las que no." En otro caso, otro participante respondió al mensaje incluyendo la frase "Tendría algunas sugerencias para la carrera". Si encontramos que dos de ellos lo expresan de forma voluntaria, y lo hacen posteriormente al llenado de la encuesta, significa que debe haber un número mayor de profesores egresados que tiene cosas para decir de esta temática, y evidentemente, se encuentra con que no existen los canales apropiados para hacerlo. El simple llenado de una encuesta requerida por un investigador, propició intervenciones como las descritas anteriormente.

\section{Análisis general}

Lo primero que se realizó con la serie de respuestas es encontrar la valoración media entre 0 y 5 , donde se asigna al valor 2,5 el límite entre una valoración positiva y una negativa. Una vez realizadas las valoraciones medias y ordenados de menor a mayor, se obtienen los datos observados en la Figura $\mathrm{N}^{\circ} 1$. Se constata que 13 de 37 materias (35\%) tienen coeficientes menores de 2,5 y por tanto el $65 \%$ (casi dos tercios) tienen valoraciones consideradas positivas $(>2,5)$.

\begin{tabular}{c|r|}
\hline Sem. Ed Sex & 1,321 \\
\hline Inglés/Lenguas extr & 1,469 \\
\hline Sem. DDHH & 1,513 \\
\hline Hist de la Educación & 1,629 \\
\hline Sociología I & 1,721 \\
\hline Sociología de la Ed & 1,897 \\
\hline Obs. YAn. I Ed & 1,986 \\
\hline Pedagogía I & 2,059 \\
\hline Pedagogía II & 2,107 \\
\hline ECI & 2,113 \\
\hline Informática & 2,157 \\
\hline Lengua/ld. Español & 2,165 \\
\hline Inv. Educativa & 2,326 \\
\hline
\end{tabular}

\begin{tabular}{|c|r|}
\hline Filosofia Ed & 2,5 \\
\hline Sem. Dif Aprendizaje & 2,567 \\
\hline Teo Conoc y Epistem & 2,61 \\
\hline Legislación y Adm Ens & 2,679 \\
\hline Proyecto Int. & 2,73 \\
\hline Ps. Aprendizaj II & 3,056 \\
\hline Psicologia Evolutiva I & 3,104 \\
\hline Int. Didáctica & 3,157 \\
\hline Taller Laboratorio & 3,473 \\
\hline Matemática III & 3,501 \\
\hline Física Térmica & 3,519 \\
\hline Moderna & 3,579 \\
\hline
\end{tabular}

\begin{tabular}{|c|r|}
\hline Matemática II & 3,596 \\
\hline Electromagnetismo & 3,696 \\
\hline Física Exp I & 3,709 \\
\hline Matemática I & 3,794 \\
\hline Ondas yóptica & 3,822 \\
\hline Fisica Exp II & 3,873 \\
\hline Mecánica & 4,087 \\
\hline Fisica II & 4,301 \\
\hline Didáctica 1 y PD & 4,348 \\
\hline Física I & 4,485 \\
\hline Didáctica 2 y PD & 4,508 \\
\hline Didáctica 3 y PD & 4,826 \\
\hline
\end{tabular}

Figura 1: Tabla con las valoraciones medias de cada asignatura, ordenadas de menor a mayor. En la columna de la izquierda se observa la lista con valoraciones menores a 2,5. La columna central y la de la derecha contienen las asignaturas con valoraciones positivas.

Se puede observar la misma información en forma gráfica, como se presenta en la Figura $\mathrm{N}^{\circ}$ 2. La línea punteada vertical separa a las asignaturas con un coeficiente mayor a 2,5 de las que tuvieron un coeficiente menor. En el eje horizontal están etiquetadas cada una de las asignaturas. 


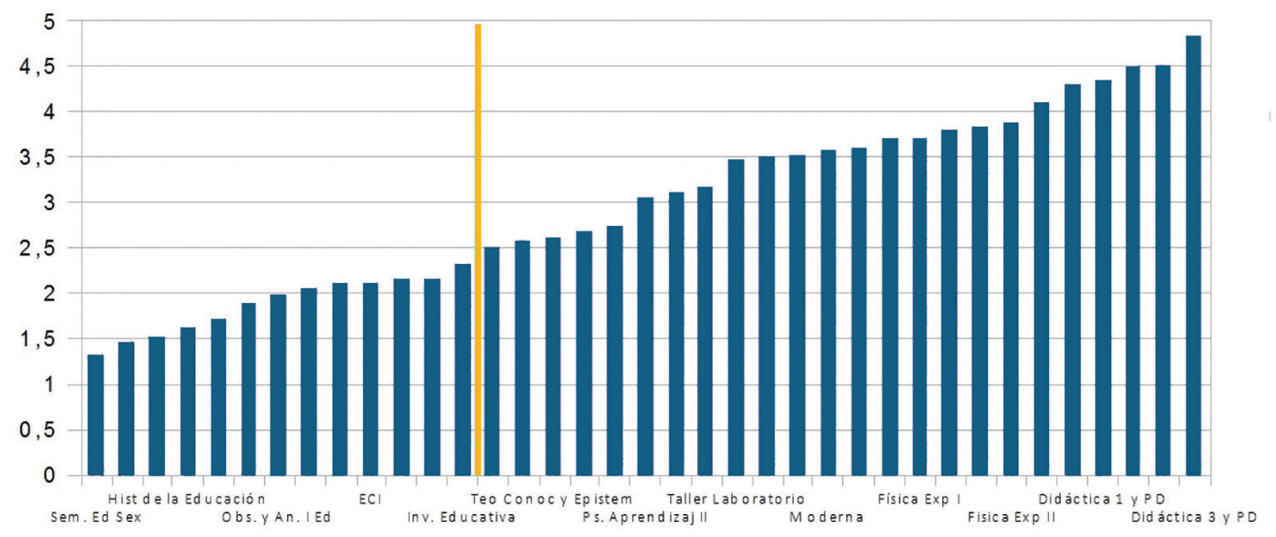

Figura 2: Ordenamiento de las asignaturas en forma gráfica. La línea punteada vertical separa las asignaturas con valoraciones mayores o menores de 2,5

Si se realiza un análisis por cada año de la carrera, se observa que no hay una diferencia significativa en el promedio de las asignaturas entre los diferentes años, como se aprecia en la Figura 3.

\begin{tabular}{|c|c|}
\hline Prom. Asignaturas 1ำ & 2,829 \\
\hline Prom. Asignaturas 2ำ & 3,016 \\
\hline Prom. Asignaturas 3ำ & 2,968 \\
\hline Prom. Asignaturas 4으 & 3,09 \\
\hline
\end{tabular}

Figura 3: Promedio de valoración por año lectivo.

Análisis de casos de materias en particular

1. El curso de Física I tiene coeficiente más alto en comparación a Física Experimental I, de la misma forma en que Física II en comparación con Física Experimental II. Es un problema ya investigado, globalmente y también en Uruguay. Se puede resumir esa problemática con las palabras de Yoldi y Suárez (2014, 125): "El supuesto rol motivador que debiera tener el trabajo de laboratorio en una ciencia de base experimental como la Física, en general no es tal, ni tampoco es un puente para desarrollar en los estudiantes un aprendizaje significativo, ni destrezas cognitivas de alto nivel". El coeficiente de estas asignaturas experimentales es inclusive menor que el de Matemática I. Cabe preguntarse si esto es causa o consecuencia de los problemas recurrentes de la enseñanza de la Física experimental. ¿O quizá una mezcla de ambas?

2. Dentro del grupo de asignaturas con coeficientes menores a 2,5, se encuentra una asignatura específica: Espacio Interdisciplinario (E.C.I). Si se analizan los datos originales mostrados en la Figura 4, se observa además una alta dispersión. Cabe aquí un mayor análisis de lo que sucede en este caso, porque es una asignatura sin programa prescrito, sino que la sala local es la que lo define, dentro de un conjunto de propuestas. Es por tanto necesario revisar si este mecanismo es el adecuado, amén de discutir objetivos y posibles contenidos.

3. Era previsible que Física I y II tuvieran los valores más altos dentro de las específicas porque la distancia entre los conocimientos aprendidos y los contenidos impartidos en enseñanza media es menor que en las materias de profundización. Aun así, cabe destacar que no se percibe que en las respuestas de los encuestados haya permeado la idea de una "utilidad temática", es decir, medir la asignatura por la mera cercanía entre los temas tratados y lo que se 
va a enseñar en las aulas de enseñanza media. En ese caso, es interesante ver los valores altos de materias como Moderna $(3,822)$ u Ondas $(3,579)$, cuyo temario es muy escasamente trabajado en los cursos actuales del C.E.S. Por lo tanto, la hipótesis de que los egresados percibieron el impacto de las asignaturas principalmente en función de los contenidos temáticos tratados en cada curso se diluye. Lo mismo puede verse en los coeficientes obtenidos en los tres cursos de Matemática (3,794; 3,596 y 3,501 respectivamente). Esto es destacable porque, de alguna manera, el egresado no se ve a si mismo como un aplicador de lo aprendido en el profesorado, es decir lejos de la racionalidad tecnocrática donde el docente se limita seguir las estrategias prescritas por grupos de expertos. Es posible entonces imaginarse docentes reflexivos sobre las decisiones sobre qué y cómo enseñar. Por otra parte puede interpretarse que el docente se siente cada vez más sólido con las herramientas conceptuales que va adquiriendo, herramientas que implican conocimientos en rangos mas amplios que los necesarios para dictar una clase de enseñanza media.

\begin{tabular}{|ccccccc|}
\hline & $\mathbf{0}$ & $\mathbf{1}$ & $\mathbf{2}$ & $\mathbf{3}$ & $\mathbf{4}$ & $\mathbf{5}$ \\
$\mathrm{ECl}$ & $21,7 \%$ & $13,3 \%$ & $18,3 \%$ & $28,3 \%$ & $15,0 \%$ & $3,3 \%$ \\
\hline
\end{tabular}

Figura 4: Datos discriminados para ECI.

4. Introducción a la Didáctica tiene un coeficiente bastante más bajo que el resto de los cursos del área, lo que era previsible dada la desconexión de ese curso con la práctica docente. Se refuerza entonces la idea que didáctica y práctica docente deben formar una unidad.

5. En el caso de los cursos de Didáctica con Práctica Docente, (Didáctica I, II y III) son los cursos con mayor coeficiente, y además con una convergencia en las opiniones, registrándose poca dispersión, como se observa en la Figura 5. En ninguno de los tres casos hay opiniones en la franja de 0 y 1 . Esto condice con lo planteado por Davini $(2016,144)$ en cuanto a la gradualidad del proceso de formación en las prácticas. En ese sentido, la autora plantea como estrategia más adecuada que la residencia, o práctica docente autónoma sea en $4^{\circ}$ año, dejando para el $2^{\circ}$ y $3^{\circ}$ año instancias de trabajo con docentes experimentados, que Davini describe, entre otros dispositivos, como "demostración crítica” (2016, 140), rol que en el plan 2008 lo cumplen los profesores adscriptores. Asimismo, y unido al punto 4, Davini plantea para el primer año dispositivos como pasantías de observación, participación y trabajos de campo.

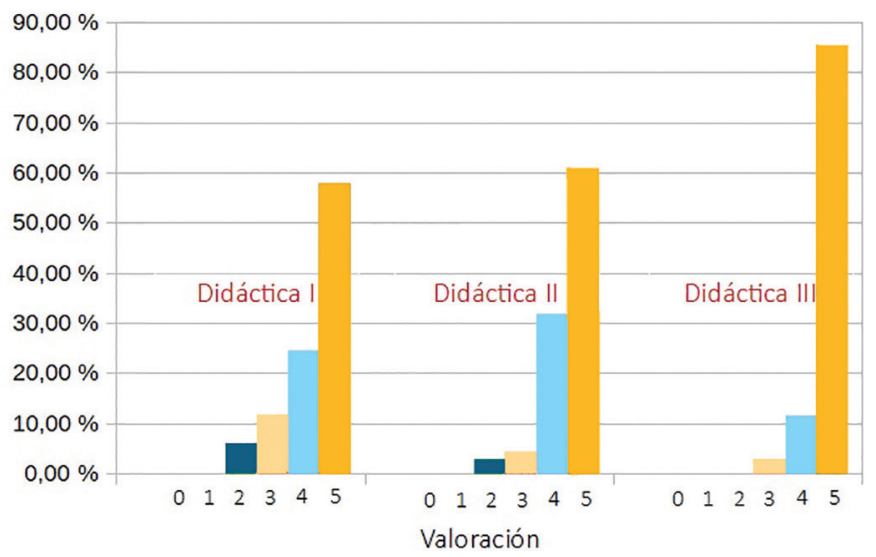

Figura 5: Gráfica conjunta de la valoración de los cursos de Didáctica I II y III, que tienen práctica. 
El bajo índice de Investigación educativa $(2,326)$ es llamativo, porque si bien en el apartado 3 se menciona la imagen de un docente que puede no identificarse como mero aplicador y sí como reflexivo sobre las decisiones sobre lo que enseñar y cómo hacerlo, no parece percibir, según esta valoración, la importancia de la investigación sistemática en esa reflexión. Eso se afirma aún más si se tiene en cuenta que esta asignatura se ubica en el tercer año de la carrera, donde el estudiante ya ha pasado por otras asignaturas de reflexión pedagógica, donde de alguna manera ya ha tomado contacto con la importancia de las investigaciones en el campo educativo.

6. Unido a Investigación educativa está el bajo coeficiente de Inglés -lenguas extranjeras- (1,469), donde el $77 \%$ de los docentes puntuaron entre 0 y 2. Esto podría deberse a que, por un lado, haya alumnos que ya hayan estudiado idiomas antes y por tanto el curso no les aportó conocimiento, o por otro lado, que no se perciba aún la importancia de leer las investigaciones, particularmente las relativas a la enseñanza de la Física que se publican en inglés. El alto volumen de producción de investigación en enseñanza de la Física derivado de lo que se denomina PhysicsEducationResearch (P.E.R.) ${ }^{7}$, que está escrito en inglés, es clave para la mejora de las clases. Por tanto, aquí hay otro punto que debe ser analizado con mayor profundidad.

7. Resultó también llamativamente bajo el coeficiente de los seminarios de de Educación Sexual $(1,321)$ y el seminario de Derechos Humanos $(1,513)$. En ninguno de los dos casos tienen opiniones que los hayan calificado con 5 . Cabe preguntarse aquí si será a causa de la temática, pues como se planteó anteriormente no puede asignarse la razón exclusiva a alguna mala práctica docente coyuntural. Como preguntas orientadoras de futuras indagaciones podría plantearse: ¿El profesor de Física se siente alejado de las situaciones que puedan surgir en el aula en relación a la educación sexual o a los derechos humanos? ¿O estos temas no forman parte de una preocupación general del cuerpo docente, no solo de los profesores de Física? Si por el contrario, la razón principal de la baja valoración fuera el poco tiempo destinado al seminario, el correspondiente a Dificultades de Aprendizaje debería haber tenido también baja valoración, pero sin embargo éste fue mayor a 2,5.

8. Los conocimientos sobre Psicología, tanto evolutiva como de aprendizaje, es visto como clave en la realidad del aula, siendo las dos asignaturas del tronco común que tienen los coeficientes más altos.

9. En el caso de epistemología, si bien el promedio es mayor a 2,5 (2,61), presenta casi un $40 \%$ de las opiniones entre 0 y 2 puntos, como se percibe en la Figura 6.

\begin{tabular}{|c|c|c|c|c|c|c|}
\hline & $\mathbf{0}$ & $\mathbf{1}$ & $\mathbf{2}$ & $\mathbf{3}$ & $\mathbf{4}$ & $\mathbf{5}$ \\
\hline Teo Conocy $y$ Epistem & $10,60 \%$ & $9,10 \%$ & $19,70 \%$ & $36,40 \%$ & $18,20 \%$ & $6,10 \%$ \\
\hline
\end{tabular}

Figura 6: Datos discriminados para Teoría del Conocimiento y Epistemología

10. Se debería analizar con detenimiento, porque los contenidos de la asignatura son, a priori, uno de los más "cercanos" a la Física de todas las materias que componen el núcleo de formación común. Por ejemplo, Kuhn era Doctor en Física de la Universidad de Harvard, Popper daba clases de Física en la universidad, y MoritzSchlick, el fundador del Círculo de Viena estudió Físi-

$7 \quad$ Accesible en sitios abiertos como physport.org o compadre.org. 
ca en Berlín con Planck. Por otra parte, es de gran importancia la reflexión epistemológica sobre lo que se enseña. En el plan 1986 esta asignatura estaba en $4^{\circ}$ año, en vez de $2^{\circ}$, ¿no será que tiene mayor impacto el estudio de esta temática cuando se tiene otro recorrido por ejemplo en la reflexión didáctica?

\section{Proyecciones: La propuesta de elaboración del índice de presencia}

La construcción de este índice se realizará con el propósito principal de establecer un coeficiente de presencia de los estudiantes de profesorado que dictan clases en las instituciones de enseñanza media del Uruguay. Con este dato cuantitativo, es posible mapear el territorio de modo de poder conocer la evolución del impacto de los diferentes centros y modalidades de formación. Si se toman a los estudiantes que están dictando clases, a partir de sus escolaridades se puede observar la evolución del impacto del profesorado más allá de solo contabilizar la cantidad de egresados. Los estudiantes que dan clases en las instituciones públicas son un porcentaje significativo. De hecho, en esta misma encuesta el $87 \%$ manifestó haber trabajado como docente antes de egresar.

En concreto, lo primero que se hace es normalizar en base 100 el impacto de cada asignatura, es decir corregir los índices de modo que la suma de todas las materias sea 100. Como se percibe en la Figura 7, la suma de los coeficientes obtenidos de la encuesta de valoración entre 0 y 5 es 109,983. Si se normalizan esos valores, tomando como 100 la suma de todos los coeficientes se puede realizar un impacto en términos de porcentaje de incidencia. La segunda columna indica los valores normalizados en base 100 , tomados como máximo con dos decimales.

\begin{tabular}{|c|r|r|}
\hline Sem. Ed Sex & 1,321 & 1,2 \\
\cline { 1 - 1 } Inglés/Lenguas extr & 1,469 & 1,34 \\
\cline { 1 - 1 } Sem. DDHH & 1,513 & 1,38 \\
\cline { 1 - 1 } Hist de la Educación & 1,629 & 1,48 \\
\cline { 1 - 1 } Sociología I & 1,721 & 1,56 \\
\hline Sociología de la Ed & 1,897 & 1,72 \\
\cline { 1 - 1 } Obs. YAn. I Ed & 1,986 & 1,81 \\
\cline { 1 - 1 } Pedagogía I & 2,059 & 1,87 \\
\hline Pedagogía II & 2,107 & 1,92 \\
\hline ECI & 2,113 & 1,92 \\
\hline Informática & 2,157 & 1,96 \\
\hline Lengua/Id. Español & 2,165 & 1,97 \\
\hline Inv. Educativa & 2,326 & 2,12 \\
\hline
\end{tabular}

\begin{tabular}{|c|r|r|}
\hline Filosofía Ed & 2,5 & 2,27 \\
\hline Sem. Dif Aprendizaje & 2,567 & 2,33 \\
\hline Teo Conoc y Epistem & 2,61 & 2,37 \\
\hline Legislación y Adm Ens & 2,679 & 2,44 \\
\hline Proyecto Int. & 2,73 & 2,48 \\
\hline Ps.Aprendizaj II & 3,056 & 2,78 \\
\hline Psicologia Evolutiva I & 3,104 & 2,82 \\
\hline Int. Didáctica & 3,157 & 2,87 \\
\hline Taller Laboratorio & 3,473 & 3,16 \\
\hline Matemática III & 3,501 & 3,18 \\
\hline Física Térmica & 3,519 & 3,2 \\
\hline Moderna & 3,579 & 3,25 \\
\hline
\end{tabular}

\begin{tabular}{|c|r|r|}
\hline Matemática II & 3,596 & 3,27 \\
\cline { 1 - 1 } Electromagnetismo & 3,696 & 3,36 \\
\cline { 1 - 1 } Física Exp I & 3,709 & 3,37 \\
\cline { 1 - 1 } Matemática I & 3,794 & 3,45 \\
\hline Ondas yóptica & 3,822 & 3,48 \\
\hline Fisica Exp II & 3,873 & 3,52 \\
\hline Mecánica & 4,087 & 3,72 \\
\hline Fisica II & 4,301 & 3,91 \\
\hline Didáctica 1 y PD & 4,348 & 3,95 \\
\hline Física I & 4,485 & 4,08 \\
\hline Didáctica 2 y PD & 4,508 & 4,1 \\
\hline Didáctica 3 y PD & 4,826 & 4,39 \\
\hline
\end{tabular}

Figura 7: Para cada asignatura se establecen dos números: la valoración media (entre 0 y 5 , ya mostrada, que fue obtenida del promedio de la encuesta) y a la derecha el coeficiente normalizado en base 100, para el cálculo del indice de impacto.

Se plantea el siguiente ejemplo de utilización del índice de presencia: se supondrá que se trata de un alumno que ha cursado los 4 años de la carrera, pero para egresar aún debe rendir 5 asignaturas. Para el ejemplo, supondremos que no ha aprobado Historia de la Educación $(1,48)$, Física Térmica $(3,2)$, Física Moderna $(3,25)$, Ondas $(3,48)$ y Legislación de la enseñanza $(2,44)$. Entre paréntesis se ha colocado el valor del coeficiente normalizado (base 100) de cada una de las asignaturas que el alumno del ejemplo aún no ha rendido. Por tanto, lo que le falta por aprobar corresponde a 13,85. Es decir, que su índice de impacto es $100-$ $13,85=86,15$. Esto permitiría no solo mapear geográficamente a los estudiantes 
que están trabajando actualmente en el sistema público, con el grado de avance correspondiente de la carrera, sino también hacer un seguimiento a través del tiempo, para ver si hay aumento, disminución o estancamiento en el coeficiente general de aquellos casos con la carrera incompleta, o variaciones por región. Así se puede, por un lado, valorar el impacto de que los estudiantes de profesorado tengan a cargo los cursos de enseñanza media en aquellos lugares donde no hay docentes titulados, y por otro lado, detectar aquellos lugares - y a aquellos docentes sin la carrera completa - donde es necesaria una acción proactiva por parte del CFE en coordinación con CETP ${ }^{8}$ y CES para promover el avance en la carrera y finalmente la imprescindible titulación.

Se propone esta manera de medir el impacto, pues considerar que hacer un índice por créditos conseguidos o materias aprobadas da una mirada incompleta del impacto de la formación en las aulas de enseñanza media y que esta ponderación construida a partir de la encuesta realizada a los egresados nóveles, brinda a este índice un mayor grado de fiabilidad. La congruencia de las respuestas obtenidas haciendo cortes a las 20 y 45 respuestas, da mayor fortaleza a esta hipótesis. 


\section{Referencias}

CES (Consejo de Educación Secundaria, 2018), "Acta 53, Resolución 64" del 8 de octubre de 2018.

Davini, Mo Cristina (2016), "La formación en la práctica docente", Ed. Paidos, Bs. As

Klein, Gustavo (2015). “El educador en Física en CES Libro II”, Trabajo realizado como investigación en las horas de Departamento del CFE, 2014-2015, Montevideo.

Parrella, Alejandro (2018) "Impacto de la modalidad semipresencial en la formación de docentes de Física en el Uruguay en términos de equidad “. Tesis de Maestría en Educación, Sociedad y Política. A probada el 22 de octubre de 2018. FLACSO Uruguay.

Presidencia de la República, 28 de noviembre de 2017. "Más del $60 \%$ de los alumnos de formación docente cursa sus estudios en el interior del país" Entrevista a Mag. Ana Lopater, directora general del CFE. Recuperado de https://presidencia. portal.gub.uy/wps/wcm/connect/presidencia/portalpresidencia/ comunicacion/comunicacionnoticias/educacion-formacion-docentelopater-alumnos-interior-cfe. Último acceso 23 de abril de 2019.

Yoldi, A. y Suárez, A (2014), "Una mirada uruguaya al trabajo experimental en la enseñanza de la Física: En la búsqueda de nuevas perspectivas”, Revista do IMEA-UNILA, Vol. 2, Num. 2, p. 123-131. Recuperado de https://revistas.unila.edu.br/ IMEA-UNILA/article/view/347 Acceso: 14 de abril de 2019.

\section{Agradecimientos}

- A todos los profesores egresados que voluntaria y generosamente participaron de esta encuesta.

- Para poder contactar a los egresados de todo el país, fue imprescindible la colaboración de muchos compañeros que, desinteresadamente, facilitaron muchos datos. En orden alfabético, muchas gracias a Cristina Araújo, Daniel Barone, Marcelo Berruti, Jimena Campaña, Javier Carro, Jose Luis Di Laccio, Rosmary Fajardo, Elizabeth Flores, Gustavo Klein, Jorge Martins, Patricia Nizarala, Shirley Pérez, Ruben Rodríguez, Julio Rolón, Andrea Seijas, Gabriela Tomás, Patricia Urruzmendi, Marie Noel Vallo, Silvia Villalpando y Eduardo Würth.

- También un especial agradecimiento a las profesoras Raquel Escudero, Andrea Seijas, Andrea Viana y Jimena Zorrilla que participaron generosamente del testeo inicial del instrumento, y que con sus comentarios permitieron ajustarlo y mejorarlo. 